\title{
NON-UNIQUENESS FOR PLATEAU'S PROBLEM. A BIFURCATION PROCESS
}

\author{
JOHANNES C. C. NITSCHE
}

The discussion to follow will deal with aspects of Plateau's problem about which no exhaustive information is available today, and particular attention will be paid to the phenomenon of non-uniqueness. It is well known that the area of a minimal surface, its suggestive name notwithstanding, need not furnish a minimum (absolute or relative) among the areas of all surfaces having the same boundary. Let us consider a minimal surface $S=\left\{\mathfrak{x}=\mathfrak{r}(u, v) ; u^{2}+v^{2}<R^{2}\right\}$ which lies imbedded in Euclidean 3-space. We shall assume that $u$ and $v$ are isothermal parameters on $S$ so that $\mathfrak{r}_{u}^{2}(u, v)=\mathfrak{r}_{v}^{2}(u, v) \equiv E(u, v)>0$ and $\mathfrak{r}_{u}(u, v) \mathfrak{r}(u, v) \equiv F(u, v)=0$. Let us further denote by $\mathfrak{X}(u, v)$ the unit normal vector of our surface and by $K(u, v)$ its Gaussian curvature. On $S$ we consider the one-parameter family of Jordan curves

$$
\Gamma_{r}=\{\mathfrak{r}=\mathfrak{r}(r \cos \vartheta, r \sin \vartheta) ; 0 \leqq \vartheta \leqq 2 \pi\}, \quad 0<r<R,
$$

which bound expanding portions

$$
S(r)=\left\{\mathfrak{r}=\mathfrak{r}(u, v) ;(u, v) \in \bar{P}_{,}\right\}
$$

of $S$. Here $\bar{P}_{q}$ is used as an abbreviation for the closure of the disc $P_{*}=\left\{u, v ; u^{2}+v^{2}<r^{2}\right\}$. For sufficiently small values of $r$ two assertions are valid:

i) $S(r)$ is the unique solution of Plateau's problem for $\Gamma_{q}$.

ii) The area $A[S(r)]$ of $S(r)$ furnishes the absolute minimum among the areas of all disc-type surfaces bounded by $\Gamma_{r}$.

As $r$ increases, either one of these statements may become false. As for i), $S(r)$ will remain unique as long as $\Gamma_{\gamma}$ retains a simply covered convex curve as its parallel, or central, projection on a plane (see [10]); or as long as the total curvature of $\Gamma_{r}$ does not exceed the value $4 \pi$ (see [7]). On the other hand, the minimizing property of the surface $S(r)-$ more precisely, the question whether $A[S(r)]$ remains at least a relative 
minimum - depends on the second variation of its area which, in turn, is closely related to the eigenvalue problem

$$
\Delta \xi-2 \lambda E K \xi=0 \quad \text { in } P_{r}, \quad \xi=0 \text { on } \partial P_{r} .
$$

Because $K(u, v)$ is non-positive and vanishes at most in isolated points, this problem will have a sequence of eigenvalues $\left\{\lambda_{n}(r)\right\}$ satisfying the inequalities $0<\lambda_{1}(r)<\lambda_{2}(r) \leqq \lambda_{3}(r) \leqq \ldots$ and corresponding eigenfunctions $\xi_{1}(u, v ; r), \xi_{2}(u, v ; r), \ldots$ subject to the ortho-normality relations

$$
2 \int_{P_{r}} \int_{E} E|K| \xi_{m} \xi_{n} d u d v=\delta_{m n} .
$$

The smallest eigenvalue $\lambda_{1}(r)$ is a continuous and strictly decreasing function of $r$.

If $\eta(u, v)=\sum_{n=1}^{\infty} c_{n} \xi_{n}(u, v ; r)$ denotes an arbitrary regular function, vanishing on $\partial P_{r}$, then the area of the comparison surface $S^{(\varepsilon)}(r)=$ $\left\{\mathfrak{r}=\mathfrak{r}(u, v)+\varepsilon \eta(u, v) \mathfrak{X}(u, v) ;(u, v) \in \bar{P}_{y}\right\}$ is found to be

$$
\begin{aligned}
A\left[S^{(\varepsilon)}(r)\right] & =A[S(r)]+\frac{1}{2} \varepsilon^{2} \int_{P_{r}}\left(\eta_{u}^{2}+\eta_{v}^{2}+2 E K \eta^{2}\right) d u d v+O\left(\varepsilon^{3}\right) \\
& =A[S(r)]+\frac{1}{2} \varepsilon^{2} \sum_{n=1}^{\infty}\left(\lambda_{n}(r)-1\right) c_{n}^{2}+O\left(\varepsilon^{3}\right) \\
& \geqq A[S(r)]+\varepsilon^{2}\left(\lambda_{1}(r)-1\right) \iint_{P_{r}} E|K| \eta^{2} d u d v+O\left(\varepsilon^{3}\right) .
\end{aligned}
$$

If $\lambda_{1}(r)>1$, then $S(r)$ can be imbedded in a field of minimal surfaces (a detailed construction can be found in [7]), and by a classical argument going back to H. A. Schwarz ([11], pp. 224-240,332-334) it is seen that the area of $S(r)$ furnishes a strong relative minimum. Moreover, since the boundary value problem $\Delta \eta-2 E K \eta=0$ in $P_{,}, \eta=0$ on $\partial P_{q}$ under the assumption $\lambda_{1}(r)>1$ has the unique solution $\eta(u, v) \equiv 0$, it follows (see e.g. [2], Lemma 3.4) that $S(r)$ is isolated or, as we shall also say, locally unique; see [8]. By [1] the inequality $\lambda_{1}(r)$ is guaranteed at least as long as the total curvature $\iint_{P_{r}} E|K| d u d v$ of $S(r)$ remains smaller than $2 \pi$.

If $\lambda_{1}(r)<1$, then (by choosing $\eta(u, v)=\xi_{1}(u, v ; r)$ ) disc-type surfaces bounded by $\Gamma_{r}$ and lying arbitrarily close to $S(r)$ can be constructed whose area is smaller than the area of $S(r) . S(r)$ is thus seen to be unstable. It is a matter of record, however, that $\Gamma_{r}$ also bounds a solution surface of least area for Plateau's problem. Obviously, this surface 
must be distinct from $S(r)$. Whether it is close to $S(r)$ or not is, of course, another question. In any case, $\Gamma_{r}$ bounds at least two solutions of Plateau's problem whenever $\lambda_{1}(r)<1$.

The preceding remarks suggest the possible existence of two positive numbers $r_{1}$ and $r_{2}$ defined as follows: $r_{1}$ is the supremum of all those values $r$ for which the surface $S(r)$ represents the unique solution of Plateau's problem for $\Gamma_{r}$. The number $r_{2}$ is the supremum of all those values $r$ for which the area of $S(r)$ is a strong relative minimum among the areas of all disc-type surfaces bounded by $\Gamma_{r}$, i.e., $\lambda_{1}\left(r_{2}\right)=1$. Obviously, the inequalities $0<r_{1} \leqq r_{2}$ hold. Since it is not clear whether the local uniqueness of $S(r)$ for $\lambda_{1}(r)>1$ implies its global uniqueness, the strict inequality $r_{1}<r_{2}$ cannot be excluded apriori. Our interest here is directed not so much to this question but rather to the study of the bifurcation process in which a second or more surfaces branch off from $S(r)$ as $r$ passes through the critical value $r=r_{2}$. Considering that the treasure of general insights is still hidden today, we shall pursue this bifurcation process in detail for the case of an explicit example - Enneper's minimal surface whose position vector is given with the help of isothermal parameters $u$ and $v$ by

$$
\mathfrak{r}(u, v)=\left\{\begin{array}{c}
u+u v^{2}-\frac{1}{3} u^{3} \\
-v-u^{2} v+\frac{1}{3} v^{3} \\
u^{2}-v^{2}
\end{array}\right\} .
$$

We have $E(u, v)=\left(1+u^{2}+v^{2}\right)^{2}$ and $K(u, v)=-4\left(1+u^{2}+v^{2}\right)^{-4}$. It is known (see [9], § 90) that the part of Enneper's surface which corresponds to the disc $u^{2}+v^{2}<3$ is free of self-intersections. In particular, the image of every concentric circle $u^{2}+v^{2}=r^{2}, 0<r<\sqrt{3}$, is a Jordan curve $\Gamma_{r}=\{\mathfrak{x}=\mathfrak{z}(\vartheta ; r) ; 0 \leqq \vartheta \leqq 2 \pi\}$ with the position vector

$$
z(\vartheta ; r)=\mathfrak{r}(r \cos \vartheta, r \sin \vartheta)=\left\{\begin{array}{c}
r \cos \vartheta-\frac{1}{3} r^{3} \cos 3 \vartheta \\
-r \sin \vartheta-\frac{1}{3} r^{3} \sin 3 \vartheta \\
r^{2} \cos 2 \vartheta
\end{array}\right\} .
$$

Pictures of $\Gamma_{r}$ can be found in [9], pp. 76, 79. The points $(u= \pm \sqrt{3}$, $v=0$ ) on the circle $u^{2}+v^{2}=3$ are mapped by $\mathfrak{x}(u, v)$ into the same point $\{0,0,3\}$ in space. Accordingly, we shall choose $R=\sqrt{3}$.

The curvature of the projection $\gamma_{r}$ of $\Gamma_{r}$ onto the $(x, y)$-plane is given by

$$
\frac{\left(1+r^{2}\right)\left(1-3 r^{2}\right)+4 r^{2} \sin ^{2} 2 \vartheta}{r\left[1+2 r^{2} \cos 4 \vartheta+r^{4}\right]^{3 / 2}}
$$


$\gamma_{r}$ is a convex curve for $0<r<1 / \sqrt{3}$ so that $S(r)$ is the unique solution of Plateau's problem for $\Gamma_{r}$ if $0<r \leqq 1 / \sqrt{3}$. The second criterion for uniqueness mentioned above leads to a better result. A computation shows that the total curvature $x\left(\Gamma_{r}\right)$ of $\Gamma_{v}$ is equal to

$$
\varkappa\left(\Gamma_{r}\right)=\frac{8 r}{1+r^{2}} \frac{1}{k(r)} E(k(r)), \quad k(r)=\frac{2 r}{\sqrt{1+10 r^{2}+9 r^{4}}}
$$

and that

$$
\frac{d}{d r} \varkappa\left(\Gamma_{r}\right) \geqq 6 \pi \frac{k(r)}{1+r^{2}} .
$$

Here $E(k)=\int_{0}^{\pi / 2} \sqrt{1-k^{2} \sin ^{2} \vartheta} d \vartheta$ denotes the complete elliptic integral of the second kind. We have $x\left(\Gamma_{\eta}\right)=4 \sqrt{3} E(1 / 2)=10.167 \ldots<4 \pi$ for $r=1 / \sqrt{3}$ and $x\left(\Gamma_{r}\right)=4 \sqrt{5} E(1 / \sqrt{5})=13.369 \ldots>4 \pi$ for $r=1$. The equality $x\left(\Gamma_{r}\right)=4 \pi$ is achieved for $r=0.882$ (see [9], § 828). It is therefore certain that $r_{1} \geqq 0.882$, although we conjecture that $r_{1}=1$.

The function $\zeta(u, v)=\left(1-u^{2}-v^{2}\right) /\left(1+u^{2}+v^{2}\right)$, which is positive for $u^{2}+v^{2}<1$ and zero for $u^{2}+v^{2}=1$, is a solution of the differential equation

$$
\Delta \zeta-2 E K \zeta \equiv \Delta \zeta+\frac{8}{\left(1+u^{2}+v^{2}\right)^{2}} \zeta=0 .
$$

From this fact it can be concluded that $\lambda_{1}(1)=1$ so that $r_{2}=1$. Further properties of the curves $\Gamma_{r}$ are discussed in [9], $\S 91,390-396$. It has been proved in [4] that $\Gamma_{r}$ bounds three distinct solutions of Plateau's problem for $r$ in a certain interval $r_{0}<r<\sqrt{3}$. (A crude estimate for $r_{0}$ is $r_{0} \leqq 1.682$.) From the following developments it will be seen that

as $r$ passes increasingly through the critical value $r=1$, the curves $\Gamma_{r}$ acquire the capability of bounding, in addition to Enneper's surface, two further minimal surfaces which appear in a continuous bifurcation process.

It is now necessary to interject some basic definitions. Let $\Gamma=$ $\{\mathfrak{r}=z(\tau) ; 0 \leqq \tau \leqq 2 \pi\}$ be a Jordan curve in Euclidean 3 -space. We are concerned with vectors $\mathfrak{r}(u, v)$ defined in the closure $\bar{P}$ of the unit disc $P=\left\{u, v ; u^{2}+v^{2}<1\right\}$ which map the boundary $\partial P$ onto $\Gamma$. Setting $u+i v=w=\varrho e^{i \vartheta}$ we shall henceforth interchangeably use the notations $\mathfrak{x}(u, v)$, or $\mathfrak{x}(w)$, or $\mathfrak{r}(\varrho, \vartheta)$ (and later also $\mathfrak{x}(w ; r)$ instead of $\mathfrak{r}(u, v ; r)$, etc. $)$ - whichever is most convenient. Denote by $\mathfrak{H}=\mathfrak{H}(T)$ the set of vectors $\mathfrak{r}(w) \in C^{2}(P) \cap C^{0}(\bar{P})$ which are harmonic in $P$ and which map $\partial P$ onto $\Gamma$ monotonically so that three fixed distinct points $w_{1}, w_{2}, w_{3}$ on $\partial P$ are transformed into three fixed distinct points $z_{j}=z\left(\tau_{j}\right)$, $(j=1,2,3)$, on $\Gamma$, respectively. Once and for all we shall choose $w_{1}=1$, 
$w_{2}=i, w_{3}=-1$. Then $\mathfrak{r}\left(e^{i \vartheta}\right)=\mathfrak{z}(\tau(\vartheta))$ where $\tau(\vartheta)$ is a continuous monotone function satisfying the conditions $\tau(0)=\tau_{1}, \quad \tau(\pi / 2)=\tau_{2}$, $\tau(\pi)=\tau_{3}$ and $\tau(\vartheta+2 \pi)=\tau(\vartheta)+2 \pi$. Endowed with the distance between two vectors,

$$
\left|\mathfrak{r}_{2}-\mathfrak{r}_{1}\right|=\max _{(u, v) \in \bar{P}}\left|\mathfrak{r}_{2}(u, v)-\mathfrak{r}_{1}(u, v)\right|,
$$

the set $\mathfrak{H}$ becomes a metric space. Each element $\mathfrak{r}(u, v)$ of $\mathfrak{H}$ defines a harmonic surface $S=\{\mathfrak{x}=\mathfrak{r}(u, v) ;(u, v) \in \bar{P}\}$. By definition, a solution of Plateau's problem for the curve $\Gamma$ is a harmonic surface whose position vector satisfies in $P$ the additional relations $\mathfrak{r}_{u}^{2}=\mathfrak{r}_{v}^{2}$, $\mathfrak{r}_{u} \mathfrak{r}_{v}=0$. We denote by $\mathfrak{M}$ the set of all such vectors. It is a matter of record that an element $\mathfrak{r}(u, v)$ of $\mathfrak{M}$ provides a topological mapping between $\partial P$ and $\Gamma$.

Since we shall benefit from working with the fixed parameter domain $P$, a slight change of the introductory notation is advisable: The portion of Enneper's surface bounded by $\Gamma_{\text {r }}$ will now be denoted by $S^{(0)}(r)=$ $\left\{\mathfrak{r}=\mathfrak{r}^{(0)}(w ; r) ; w \in \bar{P}\right\}$ where

$$
\mathfrak{x}^{(0)}(\varrho, \vartheta ; r)=\left\{\begin{array}{c}
r \varrho \cos \vartheta-\frac{1}{3} r^{3} \varrho^{3} \cos 3 \vartheta \\
-r \varrho \sin \vartheta-\frac{1}{3} r^{3} \varrho^{3} \sin 3 \vartheta \\
r^{2} \varrho^{2} \cos 2 \vartheta
\end{array}\right\}=\operatorname{Re}\left\{\begin{array}{c}
r w-\frac{1}{3} r^{3} w^{3} \\
i r w+i \frac{1}{3} r^{3} w^{3} \\
r^{2} w^{2}
\end{array}\right\}
$$

and $\mathfrak{r}^{(0)}(1, \vartheta ; r)=\mathfrak{z}(\vartheta ; r)$.

Let $S(r)=\{\mathfrak{r}=\mathfrak{r}(w ; r) ; w \in \bar{P}\}$ be another solution of Plateau's problem for the curve $\Gamma_{r}$. By a theorem of H. Lewy [3] the position vector of $S(r)$ is analytic in $\bar{P}$. Moreover, owing to the results of [6], the derivatives of $\mathfrak{r}(w ; r)$ can be estimated uniformly for all possible solutions. We shall refer here to the validity of the inequality

$$
\max _{0 \leqq \vartheta \leqq 2 \pi}\left\{|\mathfrak{r}(1, \vartheta ; r)|,\left|\mathfrak{r}_{\vartheta}(1, \vartheta ; r)\right|,\left|\mathfrak{r}_{\vartheta \vartheta}(1, \vartheta ; r)\right|\right\} \leqq K(r)
$$

with a universal constant $K=K(r)$ depending on $r$ alone, $0<r<\sqrt{\mathbf{3}}$. From the proofs in [6] it would not be difficult to extract an explicit expression for $K(r)$ in terms of the geometric properties of $\Gamma_{\nu}$. For the purposes at hand knowledge of the precise form of $K(r)$ would only be of minor advantage, however.

For the time being a value $r, 1 \leqq r<\sqrt{3}$ is chosen and then kept fixed. A computation shows that $\left|\mathfrak{r}_{\vartheta}^{(0)}(1, \vartheta ; r)\right|=r\left(1+r^{2}\right)$ and

$$
\left|\mathrm{r}_{\vartheta \vartheta}^{(0)}(1, \vartheta ; r)\right|=r\left[1+r^{2}\left(6+4 \cos ^{2} 2 \vartheta\right)+9 r^{4}\right]^{1 / 2} \leqq r \sqrt{1+r^{2}} \sqrt{1+9 r^{2}}
$$


From [8], p. 406, we know that $\left|\mathfrak{r}_{\vartheta}(1, \vartheta)\right| \geqq r\left(1+r^{2}\right) / 2$ as long as $\left|\mathfrak{x}-\mathfrak{r}^{(0)}\right|<\varepsilon_{1} \equiv \min (\pi / 50,1 / 175 K)$. Since $\Gamma_{r}$ is the topological image of $\partial P$ under the mapping of the position vectors of both surfaces $S^{(0)}$ and $S$, the boundary values of $\mathfrak{x}$ are related to those of $\mathfrak{x}^{(0)}$ through an expression of the form

$$
\mathfrak{r}(1, \vartheta ; r)=\mathfrak{r}^{(0)}(1, \vartheta+\lambda(\vartheta) ; r)
$$

Here $\lambda(\vartheta)$ is a periodic analytic function satisfying the inequality $\lambda^{\prime}(\vartheta) \geqq$ -1 . We write $\lambda(\vartheta)$ as a Fourier series,

$$
\lambda(\vartheta)=\frac{a_{0}}{2}+\sum_{n=1}^{\infty}\left(a_{n} \cos n \vartheta+b_{n} \sin n \vartheta\right)
$$

with the norm $\|\lambda\|$ defined by

$$
\|\lambda\|^{2}=\frac{1}{\pi} \int_{0}^{2 \pi} \lambda^{2}(\vartheta) d \vartheta=\frac{1}{2} a_{0}^{2}+\sum_{n=1}^{\infty}\left(a_{n}^{2}+b_{n}^{2}\right) .
$$

In view of the "three point condition" above, $\lambda(\vartheta)$ is subject to the conditions $\lambda(0)=\lambda(\pi / 2)=\lambda(\pi)=0$. The relation $\mathfrak{r}_{\vartheta}(1, \vartheta ; r)=$ $\left(1+\lambda^{\prime}(\vartheta)\right) \mathfrak{r}_{\vartheta}^{(0)}(1, \vartheta+\lambda(\vartheta) ; r)$ which follows from (2) implies the inequalities

$$
\frac{1}{2} \leqq 1+\lambda^{\prime}(\vartheta) \leqq \frac{1}{2} K \quad \text { whenever }\left|\mathfrak{x}-\mathfrak{r}^{(0)}\right|<\varepsilon_{1} .
$$

From [8], p. 406, we also conclude that

$$
\frac{1}{8}\left|\mathfrak{r}-\mathfrak{r}^{(0)}\right| \leqq|\lambda(\vartheta)| \leqq\left|\mathfrak{r}-\mathfrak{r}^{(0)}\right| \quad \text { for }|\lambda(\vartheta)| \leqq \frac{\pi}{3} .
$$

It follows that the size of the neighborhood of $\mathfrak{r}^{(0)}$, as measured according to the metric of $\mathfrak{H}$, is also governed by the magnitude of $L=$ $\max _{0 \leqq \vartheta \leqq 2 \pi}|\lambda(\vartheta)|$. A series expansion of (2) leads to

(3) $\mathfrak{r}(1, \vartheta ; r)=\mathfrak{r}^{(0)}(1, \vartheta ; r)+\lambda(\vartheta) \mathfrak{r}_{\vartheta}^{(0)}(1, \vartheta ; r)+\frac{1}{2} \lambda^{2}(\vartheta) \mathfrak{x}_{\vartheta \vartheta}^{(0)}(1, \vartheta ; r)+\ldots$

We denote by $\mathfrak{r}^{(k)}(\varrho, \vartheta ; r)$ the harmonic vector in $\bar{P}$ with boundary values

$$
\mathfrak{r}^{(k)}(1, \vartheta ; r)=\frac{1}{k !} \lambda^{k}(\vartheta) \frac{\partial^{k}}{\partial \vartheta^{k}} \mathfrak{r}^{(0)}(1, \vartheta ; r)
$$

The complex-valued expression

$$
\begin{aligned}
\Phi(\varrho, \vartheta)+i \Psi(\varrho, \vartheta) & =\frac{1}{2}\left(\mathfrak{r}_{\vartheta}^{2}-\varrho^{2} \mathfrak{r}_{\varrho}^{2}\right)+i \varrho \mathfrak{r}_{\varrho} \mathfrak{r}_{\vartheta} \\
& =\frac{1}{2} \varrho^{2} e^{2 i \vartheta}\left[\left(\mathfrak{r}_{v}^{2}-\mathfrak{r}_{u}^{2}\right)+i 2 \mathfrak{r}_{u} \mathfrak{r}_{v}\right]
\end{aligned}
$$


is an analytic function in $P$ for every element of $\mathfrak{H}$, i.e., for every surface whose position vector is harmonic in $P$. It is therefore easily seen that the surface $S$ is a solution of Plateau's problem if, and only if, $\Psi(1, \vartheta)=0$. From [8] we know that this equation is equivalent with the condition

$$
\sum_{m=1}^{\infty} \Lambda^{(m)}(1, \vartheta ; r, \lambda)=0
$$

Here the $\Lambda^{(m)}$ are defined by

$$
\Lambda^{(m)}(1, \vartheta ; r, \lambda)=\sum_{k=0}^{m} \frac{1}{k !} \lambda^{k}(\vartheta) \mathfrak{r}_{\varrho}^{(m-k)}(1, \vartheta ; r) \frac{\partial^{k+1}}{\partial \vartheta^{k+1}} \mathfrak{r}^{(0)}(1, \vartheta ; r) .
$$

Applying the estimating procedures used in [5], [8] we find that there are positive constants $\mathscr{C}$ and $M$ such that for $\max _{0 \leqq \vartheta \leqq 2 \pi}|\lambda(\vartheta)| \leqq L$, $\max _{0 \leqq \vartheta \leqq 2 \pi}|\bar{\lambda}(\vartheta)| \leqq L$

$$
\left\|\Lambda^{(m)}(1, \vartheta ; r, \lambda)\right\| \leqq \mathscr{C} \frac{(M L)^{m-1}}{(m-1) !}\left\|\lambda^{\prime}\right\|
$$

$$
\left\|\Lambda^{(m)}(1, \vartheta ; r, \bar{\lambda})-\Lambda^{(m)}(1, \vartheta ; r, \lambda)\right\| \leqq \mathscr{C} \frac{(M L)^{m-2}}{(m-2) !}\left(\left\|\lambda^{\prime}\right\|+\left\|\bar{\lambda}^{\prime}\right\|\right)\left\|\bar{\lambda}^{\prime}-\lambda^{\prime}\right\| .
$$

From [8], p. 408 we take over the computation of $\sigma(\vartheta ; r, \lambda) \equiv$ $\Lambda^{(1)}(1, \vartheta ; r, \lambda)$ :

$$
\begin{aligned}
\sigma(\vartheta ; r, \lambda)= & r^{2}\left(r^{2}+1\right)\left\{a_{2} c_{2}-\frac{r^{2}-1}{r^{2}+1} b_{2} s_{2}\right. \\
& \left.+\sum_{n=3}^{\infty}\left[\left(r^{2}+1\right) n-\left(3 r^{2}+1\right)\right]\left(a_{n} c_{n}+b_{n} s_{n}\right)\right\} .
\end{aligned}
$$

In this formula the abbreviations $c_{n}=\cos n \vartheta$ and $s_{n}=\sin n \vartheta$ have been used. Condition (6) can be written as

$$
\sigma(\vartheta ; r, \lambda)=-\sum_{m=2}^{\infty} \Lambda^{(m)}(1, \vartheta ; r, \lambda) \equiv \Lambda(1, \vartheta ; r, \lambda) .
$$

We turn now to the attempt to find a solution $\lambda=\lambda(\vartheta ; r)$ of $(10)$ for values $r>1$ close to $r=1$. For this purpose we set $\varepsilon=\sqrt{r^{2}-1}$ and assume $\lambda$ to be of the form

$$
\lambda(\vartheta ; r)=\varepsilon \lambda^{(1)}(\vartheta)+\varepsilon^{2} \lambda^{(2)}(\vartheta)+\ldots
$$

where

$$
\lambda^{(k)}(\vartheta)=\frac{1}{2} a_{0}^{(k)}+\sum_{n=1}^{\infty}\left(a_{n}^{(k)} \cos n \vartheta+b_{n}^{(k)} \sin n \vartheta\right)
$$


Some preparatory beuristic remarks are in order. A lengthy computation ${ }^{1}$ shows that

$$
\begin{aligned}
\Lambda^{(2)}(1, \vartheta ; r, p \sin 2 \vartheta) & =-\frac{r^{2}}{4}\left(1+14 r^{2}+3 r^{4}\right) p^{2} \sin 4 \vartheta \\
& =-\frac{9}{2} r^{2} p^{2} \sin 4 \vartheta+p^{2} O\left(\varepsilon^{2}\right) .
\end{aligned}
$$

Accordingly, we shall choose the first two terms in the expansion of $\lambda(\vartheta ; r)$ as follows:

Then

$$
\begin{aligned}
& \lambda^{(1)}(\vartheta)=b_{2}^{(1)} \sin 2 \vartheta, \\
& \lambda^{(2)}(\vartheta)=b_{2}^{(2)} \sin 2 \vartheta+b_{4}^{(2)} \sin 4 \vartheta .
\end{aligned}
$$

$$
\sigma(\vartheta ; r, \lambda)=8 \varepsilon^{2} b_{4}^{(2)} \sin 4 \vartheta+O\left(\varepsilon^{3}\right)
$$

and

$$
\Lambda(1, \vartheta ; r, \lambda)=\frac{9}{2} \varepsilon^{2}\left[b_{2}^{(1)}\right]^{2} \sin 4 \vartheta+O\left(\varepsilon^{3}\right)
$$

so that

$$
b_{4}^{(2)}=\frac{9}{16}\left[b_{2}^{(1)}\right]^{2}
$$

Now the necessity arises to compare certain terms in $\varepsilon^{3}$. For this purpose we note the following relations which can be obtained only by extensive computations (remember the abbreviations $s_{n}=\sin n \vartheta$ ):

$$
\begin{aligned}
& \Lambda^{(2)}\left(1, \vartheta ; r, p s_{2}+q s_{4}\right) \\
& =-r^{2}\left\{\frac{9}{2} p^{2} s_{4}+9 p q\left(s_{2}+s_{6}\right)+\frac{1}{16} q^{2}\left(22 s_{4}+117 s_{8}+2 s_{12}\right)\right\} \\
& \quad+p^{2} O\left(\varepsilon^{2}\right)+p q O\left(\varepsilon^{2}\right)+q^{2} O\left(\varepsilon^{2}\right) \\
& \Lambda^{(3)}\left(1, \vartheta ; r, p s_{2}\right)=r^{2} \frac{11}{6} p^{3}\left(3 s_{2}-s_{6}\right)+p^{3} O\left(\varepsilon^{2}\right) .
\end{aligned}
$$

It is now seen that the coefficients of $\sin 2 \vartheta$ are

$$
\begin{array}{ll}
\text { in } \frac{1}{r^{2}} \sigma(1, \vartheta ; r, \lambda): & -\varepsilon^{3} b_{2}^{(1)}+O\left(\varepsilon^{4}\right), \\
\text { in } \frac{1}{r^{2}} \Lambda(1, \vartheta ; r, \lambda): & 9 \varepsilon^{3} b_{2}^{(1)} b_{4}^{(2)}-\frac{11}{2} \varepsilon^{3}\left[b_{2}^{(1)}\right]^{3}+O\left(\varepsilon^{4}\right) .
\end{array}
$$

\footnotetext{
1 Unfortunately, neither this computation nor the even more extensive explicit computations, which will become necessary later on, can be reproduced here.
} 
Consequently, we shall have

$$
-b_{2}^{(1)}=9 b_{2}^{(1)} b_{4}^{(2)}-\frac{11}{2}\left[b_{2}^{(1)}\right]^{3}
$$

or, combined with (12),

$$
b_{2}^{(1)}+\left(\frac{81}{16}-\frac{11}{2}\right)\left[b_{2}^{(1)}\right]^{3}=\left(1-\frac{7}{16}\left[b_{2}^{(1)}\right]^{2}\right) b_{2}^{(1)}=0 .
$$

This condition has three distinct solutions

$$
b_{2}^{(1)}=0, \quad b_{2}^{(1)}=\frac{4}{\sqrt{7}}, \quad b_{2}^{(1)}=-\frac{4}{\sqrt{7}}
$$

and, corresponding to these solutions, $b_{4}^{(2)}=0$ in the first case and $b_{4}^{(2)}=9 / 7$ in the other two cases.

The preceding beuristic remarks indicate that a bifurcation appears as $\varepsilon$ increases from zero to small positive values. i.e., as $r$ increases from one to values larger than one. The choice $b_{2}^{(1)}=0$ leads, of course, to $\lambda(\vartheta ; r) \equiv 0$, that is to say, back to our original surface $S^{(0)}(r)$. Of the other two cases we shall now discuss rigorously the second for which $b_{2}^{(1)}=$ $4 / \sqrt{7}$. The third case can be treated similarly.

We shall try to find a solution $\lambda(\vartheta ; r)$ of $(10)$ for small positive values of $\varepsilon$ in the form

$$
\begin{aligned}
\lambda(\vartheta ; r)= & {\left[\frac{4}{\sqrt{7}} \varepsilon+\varepsilon^{2} b_{2}(\varepsilon)\right] s_{2}+\left[\frac{9}{7} \varepsilon^{2}+\varepsilon^{3} b_{4}(\varepsilon)\right] s_{4} } \\
& +\varepsilon^{3}\left\{\frac{1}{2} a_{0}(\varepsilon)+a_{1}(\varepsilon) c_{1}+b_{1}(\varepsilon) s_{1}+\mu(\vartheta ; \varepsilon)\right\}
\end{aligned}
$$

where

$$
\mu(\vartheta ; \varepsilon)=a_{2}(\varepsilon) c_{2}+a_{3}(\varepsilon) c_{3}+b_{3}(\varepsilon) s_{3}+a_{4}(\varepsilon) c_{4}+\sum_{n=5}^{\infty}\left(a_{n}(\varepsilon) c_{n}+b_{n}(\varepsilon) s_{n}\right)
$$

and the $a_{n}(\varepsilon), b_{n}(\varepsilon)$ are themselves power series in $\varepsilon$. In view of the three point condition we must have

$$
\begin{aligned}
\frac{1}{2} a_{0}(\varepsilon)+a_{1}(\varepsilon)+\mu(0 ; \varepsilon) & =0, \\
\frac{1}{2} a_{0}(\varepsilon)+b_{1}(\varepsilon)+\mu(\pi / 2 ; \varepsilon) & =0, \\
\frac{1}{2} a_{0}(\varepsilon)-a_{1}(\varepsilon)+\mu(\pi ; \varepsilon) & =0 .
\end{aligned}
$$

From these equations the three coefficients $a_{0}, a_{1}, b_{1}$ can be determined: 


$$
\begin{aligned}
a_{0}(\varepsilon)= & -2\left[a_{2}(\varepsilon)+a_{4}(\varepsilon)+a_{6}(\varepsilon)+\ldots\right] \\
a_{1}(\varepsilon)= & -\left[a_{3}(\varepsilon)+a_{5}(\varepsilon)+a_{7}(\varepsilon)+\ldots\right] \\
b_{1}(\varepsilon)= & 2\left[a_{2}(\varepsilon)+a_{6}(\varepsilon)+a_{10}(\varepsilon)+\ldots\right] \\
& +\left[b_{3}(\varepsilon)-b_{5}(\varepsilon)+b_{7}(\varepsilon)-\ldots\right]
\end{aligned}
$$

so that

$$
\frac{1}{2} a_{0}^{2}(\varepsilon)+a_{1}^{2}(\varepsilon)+b_{1}^{2}(\varepsilon) \leqq \frac{5}{2}\left\|\mu^{\prime}(\vartheta ; \varepsilon)\right\|^{2}
$$

After substitution of $\lambda(\vartheta ; r)$ from $(13),\left(1 / r^{2}\right) \Lambda(1, \vartheta ; r, \lambda)$ attains an expansion

$$
\frac{1}{r^{2}} \Lambda(1, \vartheta ; r, \lambda)=\sum_{n=2}^{\infty}\left[A_{n}(\varepsilon, \lambda) c_{n}+B_{n}(\varepsilon, \lambda) s_{n}\right] .
$$

From (5) it is clear, that neither a constant term nor terms with $\cos \vartheta$ or $\sin \vartheta$ can appear on the right hand side. In determining the coefficients $A_{n}$ and $B_{n}$ it is necessary to carry the computations to the point that explicit expressions become available for all terms up to those of order $\varepsilon^{4}$ in $B_{2}$ and all terms up to those of order $\varepsilon^{3}$ in the other coefficients. The result of these computations is as follows:

$$
\begin{aligned}
& B_{2}(\varepsilon, \lambda)=-\frac{4}{\sqrt{7}} \varepsilon^{3}+\left[\frac{180}{49}-\frac{183}{7} b_{2}(\varepsilon)+\frac{36}{\sqrt{7}} b_{4}(\varepsilon)\right] \varepsilon^{4}+\varepsilon^{5} \tilde{B}_{2}(\varepsilon, \lambda), \\
& B_{4}(\varepsilon, \lambda)=\frac{72}{7} \varepsilon^{2}+\frac{36}{\sqrt{7}} \varepsilon^{3} b_{2}(\varepsilon)+\varepsilon^{4} \tilde{B}_{4}(\varepsilon, \lambda), \\
& B_{6}(\varepsilon, \lambda)=\frac{1324}{21 \sqrt{7}} \varepsilon^{3}+\varepsilon^{4} \tilde{B}_{6}(\varepsilon, \lambda) .
\end{aligned}
$$

All the other coefficients have the form

$$
\begin{array}{ll}
A_{n}(\varepsilon, \lambda)=\varepsilon^{4} \tilde{A}_{n}(\varepsilon, \lambda), & n \geqq 2, \\
B_{n}(\varepsilon, \lambda)=\varepsilon^{4} \tilde{B}_{n}(\varepsilon, \lambda), & n \geqq 2, n \neq 2,4,6 .
\end{array}
$$

On the other hand,

$$
\begin{aligned}
\frac{1}{r^{2}} \sigma(\vartheta ; r, \lambda)=(2 & \left.+\varepsilon^{2}\right) \varepsilon^{3} a_{2}(\varepsilon) c_{2}-\left(\frac{4}{7} \varepsilon^{3}+\varepsilon^{4} b_{2}(\varepsilon)\right) s_{2} \\
& +2 \varepsilon^{3}\left(2+\varepsilon^{2}\right)\left[a_{3}(\varepsilon) c_{3}+b_{3}(\varepsilon) s_{3}\right] \\
& +\varepsilon^{3}\left(2+\varepsilon^{2}\right)\left(4+\varepsilon^{2}\right) a_{4}(\varepsilon) c_{4}
\end{aligned}
$$




$$
\begin{aligned}
& +\left(2+\varepsilon^{2}\right)\left(4+\varepsilon^{2}\right)\left(\frac{9}{7} \varepsilon^{2}+\varepsilon^{3} b_{4}(\varepsilon)\right) s_{4} \\
& +\varepsilon^{3}\left(2+\varepsilon^{2}\right) \sum_{n=5}^{\infty}\left[\left(2+\varepsilon^{2}\right) n-\left(4+3 \varepsilon^{2}\right)\right]\left(a_{n}(\varepsilon) c_{n}+b_{n}(\varepsilon) s_{n}\right) .
\end{aligned}
$$

The conditions for the coefficients $a_{0}(\varepsilon), a_{1}(\varepsilon), b_{1}(\varepsilon)$ are contained in (14). A comparison leads to a system of equations for the Fourier coefficients of $\lambda(\vartheta ; r)$. The three "special" equations are

$$
\begin{aligned}
-b_{2}(\varepsilon) & =\frac{180}{49}-\frac{183}{7} b_{2}(\varepsilon)+\frac{36}{\sqrt{7}} b_{4}(\varepsilon)+\varepsilon \tilde{B}_{2}(\varepsilon, \lambda), \\
\left(8+6 \varepsilon^{2}+\varepsilon^{4}\right) b_{4}(\varepsilon) & =\frac{36}{\sqrt{7}} b_{2}(\varepsilon)-\varepsilon\left(6+\varepsilon^{2}\right)+\varepsilon \tilde{B}_{4}(\varepsilon, \lambda) \\
\left(16+14 \varepsilon^{2}+3 \varepsilon^{4}\right) b_{6}(\varepsilon) & =\frac{1324}{21 \sqrt{7}}+\varepsilon \tilde{B}_{6}(\varepsilon, \lambda) .
\end{aligned}
$$

The remaining equations are

$$
\begin{aligned}
\left(2+\varepsilon^{2}\right) a_{2}(\varepsilon) & =\varepsilon \tilde{A}_{2}(\varepsilon, \lambda), \\
\left(2+\varepsilon^{2}\right)\left[\left(2+\varepsilon^{2}\right) n-\left(4+3 \varepsilon^{2}\right)\right] a_{n}(\varepsilon) & =\varepsilon \tilde{A}_{n}(\varepsilon, \lambda), \quad n \geqq 3, \\
\left(2+\varepsilon^{2}\right)\left[\left(2+\varepsilon^{2}\right) n-\left(4+3 \varepsilon^{2}\right)\right] b_{n}(\varepsilon) & =\varepsilon \tilde{B}_{n}(\varepsilon, \lambda), \quad n \geqq 3, n \neq 4,6 .
\end{aligned}
$$

For $\varepsilon=0$ we obtain

$$
\begin{aligned}
& a_{2}(\varepsilon)=a_{3}(\varepsilon)=\ldots=0, \\
& b_{3}(\varepsilon)=b_{5}(\varepsilon)=b_{7}(\varepsilon)=b_{8}(\varepsilon)=\ldots=0,
\end{aligned}
$$

and

$$
\begin{aligned}
-b_{2}(0) & =\frac{180}{49}-\frac{183}{7} b_{2}(0)+\frac{36}{\sqrt{7}} b_{4}(0), \\
8 b_{4}(0) & =\frac{36}{\sqrt{7}} b_{2}(0), \\
16 b_{6}(0) & =\frac{1324}{21 \sqrt{7}},
\end{aligned}
$$

or 


$$
\begin{aligned}
\frac{176}{7} b_{2}(0)-\frac{36}{\sqrt{7}} b_{4}(0) & =\frac{180}{49}, \\
-\frac{36}{\sqrt{7}} b_{2}(0)+8 b_{4}(0) & =0
\end{aligned}
$$

and $b_{6}(0)=331 /(84 \sqrt{7})$, so that

$$
b_{2}(0)=\frac{90}{49}, \quad b_{4}(0)=\frac{405}{49 \sqrt{7}}, \quad b_{6}(0)=\frac{331}{84 \sqrt{7}} .
$$

In view of the estimates (8), (15) and the solvability for $\varepsilon=0$ it can be shown that our system of equations has, for all small non-negative values of $\varepsilon$, in fact a solution $\lambda^{(+)}(\vartheta ; r)$ of the form (13) whose derivative has a uniformly bounded norm:

$$
\lambda^{(+)}(\vartheta ; r)=\varepsilon \frac{4}{\sqrt{7}} s_{2}+\varepsilon^{2}\left[\frac{90}{49} s_{2}+\frac{9}{7} s_{4}\right]+O\left(\varepsilon^{3}\right) .
$$

Let us summarize: We have seen that equations (10) have three distinct solutions for values of $r \geqq 1$ close to $r=1$ :

$$
\begin{aligned}
& \lambda^{(0)}(\vartheta ; r)=0, \\
& \lambda^{(+)}(\vartheta ; r)=\frac{4}{\sqrt{7}} \sqrt{r^{2}-1} \sin 2 \vartheta+O\left(r^{2}-1\right), \\
& \lambda^{(-)}(\vartheta ; r)=-\frac{4}{\sqrt{7}} \sqrt{r^{2}-1} \sin 2 \vartheta+O\left(r^{2}-1\right) .
\end{aligned}
$$

Each generates a solution of Plateau's problem for the Jordan curve $\Gamma_{q}$. $\lambda^{(0)}=0$ leads us back to Enneper's surface $S^{(0)}(r)$ with the position vector $\mathfrak{r}^{(0)}(\varrho, \vartheta ; r)$. But in addition to Enneper's surface two new surfaces $S^{(+)}(r)$ and $S^{(-)}(r)$ appear. In view of (3) the position vectors of these surfaces are, respectively,

$\mathfrak{r}^{( \pm)}(\varrho, \vartheta ; r)=\mathfrak{r}^{(0)}(\varrho, \vartheta ; r)+\frac{2}{\sqrt{7}} \sqrt{r^{2}-1}\left\{\begin{array}{c}\varrho^{3} c_{3}-\varrho^{5} c_{5} \\ -\varrho^{3} s_{3}-\varrho^{5} s_{5} \\ -2+2 \varrho^{4} c_{4}\end{array}\right\}+O\left(r^{2}-1\right)$.

We know that Enneper's surface is not stable for $r>1$. The surfaces $S^{(+)}(r)$ and $S^{(-)}(r)$ will be the two surfaces of least area bounded by $\Gamma_{r}$. (It is the special symmetry of $\Gamma_{r}$ which gives rise to two distinct, but congruent surfaces of least area.) While the point $u=v=0$ is mapped by $\mathfrak{r}^{(0)}$ into the origin of 3 -space for all values of $r$, its image under the mapping by the vectors $\mathfrak{r}^{(+)}$and $\mathfrak{r}^{(-)}$, roughly the point 


$$
\left(0,0,-\frac{4}{\sqrt{7}} \sqrt{r^{2}-1}\right) \text { or }\left(0,0, \frac{4}{\sqrt{7}} \sqrt{r^{2}-1}\right) \text {, }
$$

moves along the $z$-axis, down or up, as $r$ increases. In this way the surfaces $S^{(+)}$and $S^{(-)}$arrange to decrease the surface area of $S^{(0)}$.

Acknowledgement. The preceding research was supported in part by the National Science Foundation through Grant No. MPS 75-05418.

\section{References}

[1] BARbosa, J. L., and M. Do CARMo: On the size of a stable minimal surface in $R^{3}$. - Amer. J. Math. 98, 1976, 515-528.

[2] BöHмE, R., and F. TomI: Zur Struktur der Lösungsmenge des Plateauproblems. - Math. Z. 133, 1973, 1-29.

[3] LEWy, H.: On the boundary behavior of minimal surfaces. - Proc. Nat. Acad. Sci. U.S.A. 37, 1951, 103-110.

[4] NirschE, J. C. C.: Contours bounding at least three solutions of Plateau's problem. - Arch. Rational Mech. Anal. 30, 1968, 1-11.

[5] - - Concerning the isolated character of solutions of Plateau's problem. Math. Z. 109, 1969, 393-411.

[6] -》- The boundary behavior of minimal surfaces. Kellogg's theorem and branch points on the boundary. - Invent. Math. 8, 1969, 313-333.

[7] -》- A new uniqueness theorem for minimal surfaces. - Arch. Rational Mech. Anal. 52, 1973, 319-329.

[8] - - Local uniqueness for Plateau's problem. - Istituto Nazionale di Alta Matematica, Symposia Mathematica 14, Academic Press, LondonNew York, 1974, 403-411.

[9] -》- Vorlesungen über Minimalfächen. - Die Grundlehren der mathematischen Wissenschaften 199, Springer-Verlag, Berlin-HeidelbergNew York, 1975.

[10] RADó, T.: Contributions to the theory of minimal surfaces. - Acta Litt. Sci. Univ. Szeged. Sect. Sci. Math. 6, 1932, 1-20.

[11] Schwarz, H. A.: Gesammelte mathematische Abhandlungen. I. - Verlag von Julius Springer, Berlin, 1890.

University of Minnesota

School of Mathematics

Minneapolis, Minnesota 55455

USA

Received 15 August 1975 